\title{
ORBITAL ANGULAR MOMENTUM OF HIGH HARMONICS GENERATED BY A NEON JET EXCITED WITH A STRONG TWISTED LASER PULSE
}

\author{
Szilárd Majorosi ${ }^{1}$, Mihály G. Benedict ${ }^{1}$, Szabolcs Hack $^{1,2}$, Attila Czirják ${ }^{1,2}$ \\ ${ }^{1}$ Department of Theoretical Physics, University of Szeged, Tisza L. krt. 84, H-6720 Szeged, Hungary \\ ${ }^{2}$ ELI-ALPS, ELI-HU Non-Profit Ltd., Dugonics tér 13, H-6720 Szeged, Hungary
}

DOI: https://doi.org/10.14232/kvantumelektronika.9.26

\section{Introduction}

An interesting class of solutions to the Maxwell equations in the paraxial approximation are light beams with a specific amplitude distribution along their cross section in the form of a modulated Gaussian together with a twisted phase like $\mathrm{e}^{i \ell \varphi}$, when going around a circle perpendicular to the direction of propagation. These beams can be shown to carry an angular momentum $\hbar \ell$ per photon, where $\ell$ can be any integer (positive negative or zero) [1-3]. This orbital angular momentum (OAM) is diffrent from photon spin denoted usually by $\sigma$ due to possible circular polarization which is restricted to be \pm 1 or 0 .

In this contribution we present results of calculations of the spatial structure of high harmonic radiation generated from a thin gas jet of $\mathrm{Ne}$ atoms excited by a strong multicycle near-infrared pulse, carrying a low value of nonzero orbital angular momentum. The responses of the individual atoms are calculated with the 3D time dependent Schrödinger equation (TDSE), resulting in a local secondary field essentially within the sample. The amplitudes and phases of the harmonics in the far-field, i.e. on the surface of a detector have been obtained by the Fraunhofer diffraction integral. The interesting effect is that the process of harmonic generation multiplies the $\ell$ of the excitation, i.e. the OAM of the $q$-th harmonic will be $q \ell$.

\section{The mathematical formalism}

Let us first recall the mathematical form of a Laguerre-Gaussian mode (LG), which has a focus at $z=0$ :

$$
u_{p}^{\ell}(\rho, \varphi, z)=G(\rho, z) \times\left(\frac{\sqrt{2} \rho}{w(z)}\right)^{|\ell|} L_{p}^{|\ell|}\left[\frac{2 \rho^{2}}{w^{2}(z)}\right] \exp \left\{i \ell \varphi-(|\ell|+2 p) \arctan \left(\frac{z}{z_{R}}\right)\right\}
$$

where $G(\rho, z)$ is an ordinary Gaussian beam including the propagation phase factor $e^{i\left(k_{0} z-\omega t\right)}$. The beam waist $w_{0}$, the beam size $w(z)=w_{0} \sqrt{1+z^{2} / z_{R}^{2}}$, the Rayleigh length $z_{R}=k_{0} w_{0}^{2} / 2$ and the phase front radius $R(z)=\left(1+z_{R}^{2} / z^{2}\right)$ are identical with those of the Gaussian $G(\rho, z)$. There are two important differences, however. The radial amplitude distribution is modulated by the $L_{p}^{|\ell|}$ function, which is an associated Laguerre polynomial providing characteristic ring like distribution in the amplitude where the number of rings is $p+1$ for $\ell>0$. Additionally, there is also a characteristic twisted phase as shown by the factor $e^{i \ell \varphi}$ introducing a deviation from cylindrical symmetry in the amplitude, while the intensity still shows that symmetry.

Now we shall give the expression of a propagating pulse in a LG mode amplitude and phase profile. The Eq. (1) above is valid for a monochromatic carrier wave. For a pulse with a Gaussian envelope in time we shall simply take it with a delayed temporal argument $t-z / c$ and multiply it 
with the LG mode, giving the expression

$$
\mathcal{E}_{\ell, p}(\mathbf{r}, t)=F \cdot \exp \left(-2 \ln 2\left(\frac{t-t_{0}-\frac{z}{c}}{\tau}\right)^{2}\right)\left|u_{p}^{\ell}(\mathbf{r})\right| \cos \left(\omega_{0}\left(t-t_{0}\right)-\left(\arg \left(u_{p}^{\ell}(\mathbf{r})\right)+k_{0} z\right)\right),
$$

where $F$ is the maximal field strength and $\tau$ is the temporal FWHM of the pulse. Accordingly, the exciting pulse arrives at the different locations of the sample delayed or faster, as determined by the ratio $z / c$, and it has phase of a space dependent carrier envelope phase (CEP) as determined by the $\operatorname{argument}\left(\arg \left(u_{p}^{\ell}(\mathbf{r})\right)+k_{0} z\right)$ of the LG mode. Propagation effects beyond this approximation will be neglected, we assume that the form of the pulse will not be distorted when passing through the sample.

\section{Radiation of a single atom}

The reponse of the sample will be built up as the appropriate superposition of the multitude of the individual atoms, and the latter will be calculated from a quantum mechanical model. A frequently used simplification for that is the strong field approximation (SFA), as introduced by Lewenstein and coworkers [4]. It yields an approximate analytic formula for the atomic dipole moment and using further approximations (saddle point method etc.) plus with a field a Coulomb correction it provides the near-field of the radiated pattern.

In this work we go beyond this SFA model, we calculate the atomic dipole moment and its acceleration $\left(a\left(t_{1}\right)\right)$ by the numerical solution of the following 3D TDSE written in atomic units:

$$
i \frac{\partial}{\partial t_{1}} \Psi=\left[-\frac{1}{2}\left(\frac{\partial^{2}}{\partial z_{1}^{2}}+\frac{\partial^{2}}{\partial \rho_{1}^{2}}+\frac{1}{\rho_{1}} \frac{\partial}{\partial \rho_{1}}\right)-\frac{Z^{*}}{\sqrt{z_{1}^{2}+\rho_{1}^{2}}}-\mathcal{E}\left(t_{1}\right) \cdot z_{1}+V_{\operatorname{Im}}\left(\rho_{1}, z_{1}\right)\right] \Psi,
$$

where the laser field $\mathcal{E}\left(t_{1}\right)$ is taken to be linearly polarized in the $z_{1}$ direction, and the interaction is in the dipole form with the single-active-electron approximation (SAE). The outermost electron is assumed to be bound by a Coulomb potential, where the specific value we use for the effective nuclear charge: $Z^{*}=1.25929$ ensures that the ground state of this electron is equal to the first ionization energy of a $\mathrm{Ne}$ atom.

We solve (3) by our numerical method as described in [5], and we calculate the dipole acceleration as the quantum mechanical expectation value of the mean force acting on the electron according to the Ehrenfest theorem. This consists of the gradient of the atomic Coulomb potential figuring in Eq. (3) plus the effect of the external field. A macroscopic sample would require this procedure in a great number of times, using each time the exciting field at the specific location of an atom. In the expectation value the dominant part is determined by the wave function values close to the nucleus, therefore we restrict the the solutions $\Psi\left(t_{1}\right)$ to a box of size \pm 100 a.u around the center. On its border we use a complex imaginary potential (denoted by $V_{\operatorname{Im}}\left(\rho_{1}, z_{1}\right)$ ) in order to absorb the wave packets flowing out from the box.

\section{The near-field within the sample}

Now we shall consider the atomic sample as a continuous medium. The dipole acceleration $a\left(\mathbf{r}, t_{1}\right)$ due to the excitation $\mathcal{E}_{\ell, p}(\mathbf{r}, t)$ can be considered to be identical for a set of atoms within a volume element $\mathrm{d} V(\mathbf{r})$ around the spatial point $\mathbf{r}$ in the medium, as the distance between neighbouring atoms is much smaller than the characteristic length of essential changes of the exciting laser field. The field amplitude generated by the accelerated dipoles at a given frequency will be proportional to $\widetilde{a}(\mathbf{r}, \omega)$, the temporal Fourier transform of $a\left(\mathbf{r}, t_{1}\right)$. Accordingly, the near-field within the sample shall be given by $A^{(\text {near })}(\mathbf{r}, \omega) \propto \widetilde{a}(\mathbf{r}, \omega)$. In practice, this calculation is to be performed for a great number of discrete spatial points in the sample.

The procedure above is rather rigid and demanding, as the pulse shape of the excitation $\mathcal{E}_{\ell, p}(\mathbf{r}, t)$ is strictly determined in each spatial point. Therefore we have developed an efficient interpolation 
procedure to handle the problem. As we are assuming that the exciting pulse is not changed within the sufficiently thin sample, the single atom response does not depend on the spatial distribution of the atoms, therefore the spatial dependence of the excitation can be replaced by its dependence on local the peak amplitudes $F$, the CEP-s and on time $\mathcal{E}_{\ell, p}(\mathbf{r}, t) \sim \mathcal{E}(F, \mathrm{CEP} ; t)$. We calculate the single atom responses to this field strength and after the interpolation process that we perform in a way corresponding to cylindrical coordinates, we obtain a discrete spatial distribution of the near-field amplitudes, as shown in the following scheme.

$$
\mathcal{E}\left(F_{i}, \mathrm{CEP}_{\mathrm{j}} ; t\right) \underset{\begin{array}{c}
\text { single-atom } \\
\text { calculations }
\end{array}}{\longrightarrow} \widetilde{a}\left(F_{i}, \mathrm{CEP}_{\mathrm{j}} ; \omega\right) \propto A^{(\text {near })}\left(F_{i}, \mathrm{CEP}_{\mathrm{j}} ; \omega\right) \underset{\text { interpolation }}{\longrightarrow} A^{(\text {near })}(\rho, \varphi, z ; \omega) .
$$

We have used a fourth order Lagrange interpolation exploiting also the $2 \pi$ periodicity of the $\mathrm{CEP}_{j}$ variable.

The great advantage of this procedure is that after calculating $\widetilde{a}\left(F_{i}, \mathrm{CEP}_{\mathrm{j}} ; \omega\right)$ for a given temporal shape of the excitation in a sufficienty detailed manner with respect to its discrete variables, we have at hand their values for any LG beam with the same carrier frequency.

\section{Determination of the far-field at the surface of the detector}

In order to determine the radiation field that arrives to the detector, the near-field originating in the sample must be propagated until the detector. As our sample is thin with respect to the wavelength, we may assume that each harmonic with wave number $k_{q}=q k_{0}$ propagates with the speed of light. We also assume that the detector, as well as the sample is placed along the $z$ axis in a large distance compared to the size of the sample. We calculate the observed far-field with the following Fraunhofer diffraction integral over the sample volume:

$$
\begin{aligned}
A_{q}^{(\mathrm{far})}(\beta, \varphi)= & \frac{1}{\Delta Z^{\prime}} \frac{k_{q}}{2 \pi i} \frac{1}{r}\left(1+\frac{i}{k_{q} r}\right) e^{i k_{q} r} \cos \beta \iiint A_{q}^{(\text {near })}\left(\rho^{\prime}, \varphi^{\prime}, z^{\prime}\right) \rho^{\prime} \times \\
& \exp \left\{i k_{q}(1-\cos \beta) z^{\prime}-i k_{q} \rho^{\prime} \sin \beta \cos \left(\varphi-\varphi^{\prime}\right)\right\} \mathrm{d} \rho^{\prime} \mathrm{d} \varphi^{\prime} \mathrm{d} z^{\prime} .
\end{aligned}
$$

The variables $\rho^{\prime} \varphi^{\prime} z^{\prime}$ are the coordinates of the near-field in the sample, while $r$ is the distance of the detector from the center of the sample, $\varphi$ is the polar angle of the far-field coordinate and $\beta$ is the diffraction angle. We have also introduced the notation $A_{q}^{\text {(near) }}\left(\rho^{\prime}, \varphi^{\prime}, z^{\prime}\right)=A^{\text {(near) }}\left(\rho^{\prime}, \varphi^{\prime}, z^{\prime} ; q \omega_{0}\right)$. Note that the integration extends to the $z^{\prime}$ variable, too, and in order to correct for the dimension the left hand side, it is divided by the width of the sample $\Delta Z^{\prime}=z_{\max }^{\prime}-z_{\min }^{\prime}$. The term $e^{i k_{q} z^{\prime}}$ in the integrand takes into account the difference between the phases of the secondary waves within the sample.

This integral formula makes it possible to consider separately the propagation of the different harmonics originating at least in a thin sample. (In practice the resulting field may contain noninteger $q$-s, so one has to perform the sampling with respect to $q$ values.)

\section{Results}

Following the outline of the previous section, we performed the single-atom, the near-field and farfield calculations with the following configuration: we employed the $u_{0}^{1}$ LG beam with $\ell=1$ orbital angular momentum and $w_{0}=30 \mu \mathrm{m}$ beam waist, $\lambda=797.47 \mathrm{~nm}$ carrier wavelength (of period $T=$ 110 a.u.). Its peak electric intensity was $3.509 \frac{\mathrm{W}}{\mathrm{cm}^{2}}$ (corresponding to the peak electric field strength $F=0.1$ a.u.). This value was selected because it is the upper bound of the tunneling ionization regime of the single-active-electron $\mathrm{Ne}$ atom. The laser pulse was a many-cycle field with Gaussian envelope of $\tau=10$ fs FWHM (i.e. 413.414 a.u.). We placed the center of the Ne sample (gas-jet) at the beam waist, and regarded it as infinitely thin. In the single-atom calculations the integration time interval encompassed 22 field-cycles (2420 a.u.). The resolution for the interpolation: the peak intensity $F$ 

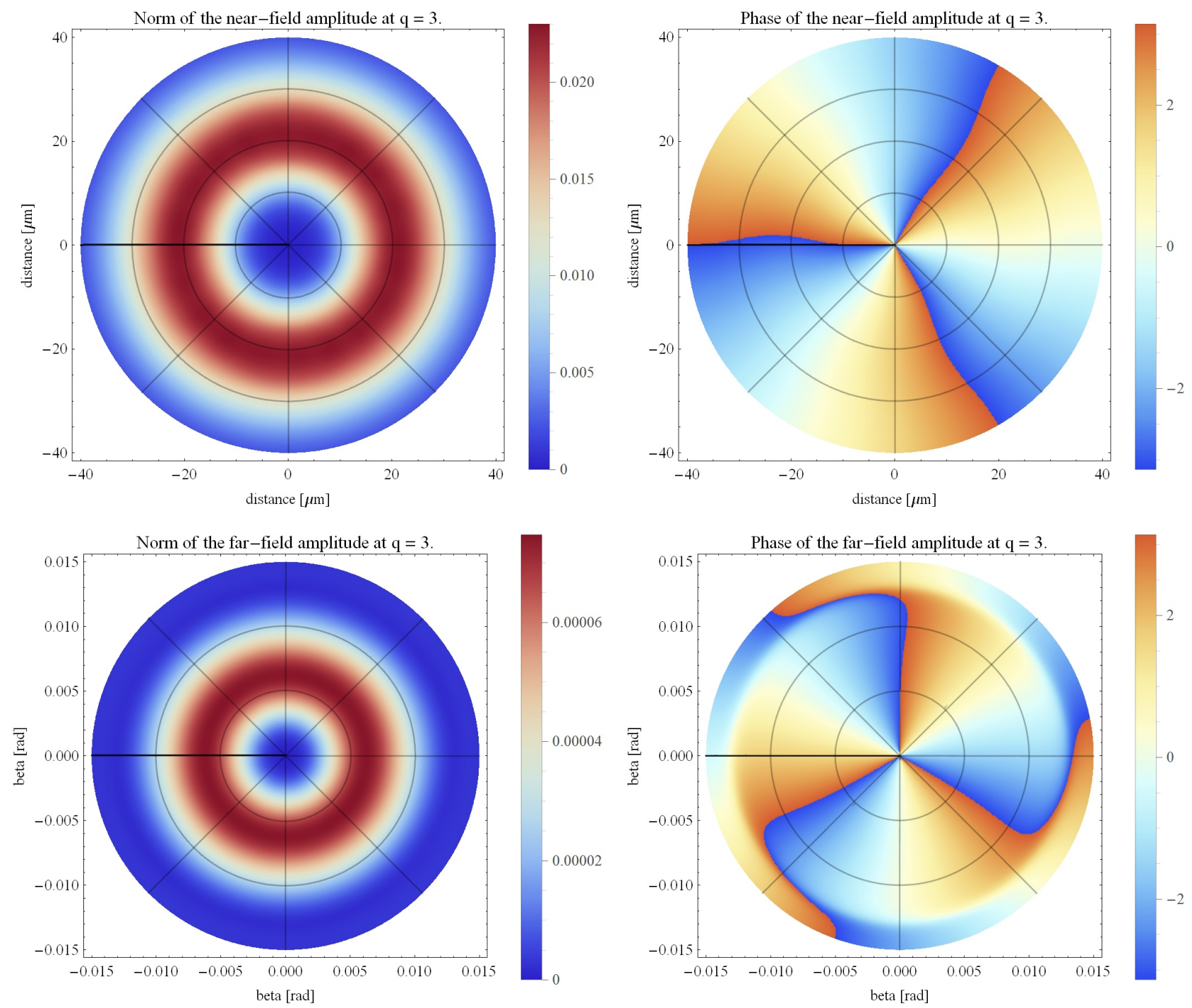

Figure 1. The spatial profiles of the $A_{q}^{(\text {near })}(\rho, \varphi, 0)$ (top) and the $A_{q}^{(\mathrm{far})}(\beta, \varphi)$ (bottom) complex electric amplitudes of the generated 3rd harmonic in the Ne sample and at the detector surface, respectively. We plot their absolute values (on the left) and phases (on the right) separately.

was sampled 21 times, and the local CEP carrier-envelope phase 80 times, that added up to 1680 single-atom calculations. We also note that we also performed the calculations of a sample of width $50 \mu \mathrm{m}$, but it failed to change the far-field amplitudes in any meaningful way.

The single-atom high-order-harmonic ( $\mathrm{HHG}$ ) spectra that we got from the integration of the full TDSE show extra detail, that the traditional SFA methods do not. These have the familiar shape of a plateau but they have a much more complicated structure compared to the ones acquired from traditional SFA (not shown here). They contain not only odd harmonics, which leads to overall delicate spatial structures that are proven to be sensitive to the local laser pulse parameters and can be different in different parts of the HHG plateau. These features have direct implications of what we see in the macroscopic sample, however.

For the spatial profiles of the near- and far-field complex electric amplitudes, we can say the following. In the traditional SFA calculations the absolute values of these amplitudes are smooth and independent from the polar angle $\varphi$, and their phase is twisted as $\ell=q \ell$ for the $q$ th harmonic. Also their HHG spectra contain only odd harmonics.

In our results, the first few harmonics up to 7th harmonic turned out to behave similarly (the 3rd 

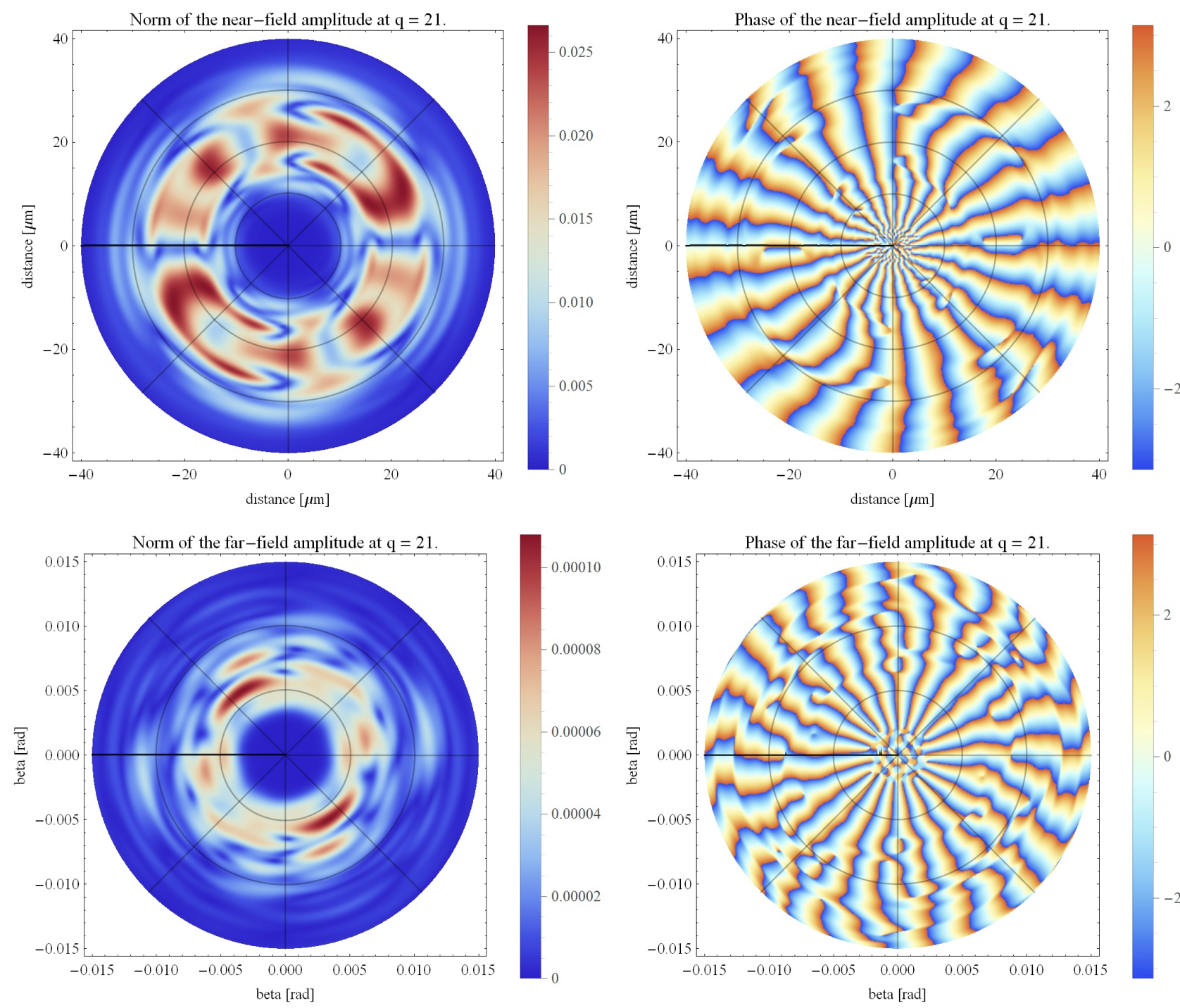

Figure 2. The spatial profiles of the $A_{q}^{(\text {near })}(\rho, \varphi, 0)$ (top) and the $A_{q}^{(\text {far })}(\beta, \varphi)$ (bottom) complex electric amplitudes of the generated 21th harmonic in the Ne sample and at the detector surface, respectively. We plot their absolute values (on the left) and phases (on the right) separately.

harmonic is shown in Figure 1). The absolute values of the near and far-fields are nearly rotationally symmetric rings, and we can see on the phase diagram that they are twisted 3 times, i.e they have a dominant orbital angual momentum of $\ell_{3}=3$ in this case. At this part of the HHG specra (which is commonly called the perturbative zone) our result are completely compliant with the traditional SFA results found in the literature. Where our method shows its merit is beyond the 7th harmonic, in the nonperturbative plateau. There, it paints a more interesting and complicated picture. To show this, we plotted the relevant near- and far-field of the generated 21th harmonic in Figure 2. The complex electric amplitude show an intricate pattern here, because the generated near-field radiation seems to be sensitive to the variations of the laser pule's local $F$ and CEP values. We can clearly see that the absolute values of the electric amplitudes clearly depend on the polar angle $\varphi$ (i.e. the local CEP value), it also creates angular zero-crossings and waves. A consequence of this is that these structures can create additional branches in the corresponding phase diagrams (by changing the sign of the field), making them more complicated compared to their SFA counterparts.

In the far-field these details are smoothed out, but they can still break the expected rotational symmetry. If we look at the $\beta$ diffraction angles where the far-field intensity peaks are located, 
their values are consistent with the existing SFA calculations (about $6 \mathrm{mrad}$ ), however they are more complicated. The far-field phase for the odd harmonics here actually are also consistent: in the ring near the maximum intensity the dominant orbital angular momentum is $\ell=21$ for the 21 th harmonic (in the corresponding figure you can count the phase shifts manually). In places where the amplitude vanishes or just very weak the angular phase is ill-defined.

In our full TDSE based results, the odd harmonics turned out to be very special in this regard, compared to the even ones: the angular phase of the latter are more disorganized, with more branches, irregularities, and no clear dominant orbital angular momentum number. Our result seems to suggest that the generated near- and far-fields contain dominantly pulses with odd angular momenta for the $\ell=1$ excitation (otherwise the multiplies of it). At frequencies which are not odd multiples of the fundamental harmonic, we saw a certain distribution of odd angular momenta (which results in an irregular phase). This would explain why the odd harmonics are special even in the full TDSE based calculations, and not contradict the SFA ones. This needs further investigation, however.

\section{Conclusions}

In this work we have computed the complex near-field and far-field electric field amplitudes of high harmonics generated in a Ne gas sample by a strong many-cycle Gaussian laser pulse. The nearfield calculation was done by numerical integration of the 3D time-dependent Schrödinger-equation in the dipole-approximation and in the single-active-electron approximation for the Ne atom. Many of these single-atom computations were performed using a pulse with local values of a LaguerreGaussian mode cross section, and then the spatial distribution of the near-field harmonic radiation was calculated by interpolation, while the corresponding far-field on a detector was determined with the Fraunhofer diffraction integral. All nonlinear propagation effects were neglected.

We found that the far-field phases of the odd harmonics were consistent with the calculations using the Lewenstein model: in the ring near the maximum intensity the dominant orbital angular momentum for the odd $q$ th harmonic turned out to be $q \ell$, while this was not true for any other harmonic. Because the actual spectra from the single-atom calculations were sensitive to the variations of the local carrier-envelope phase and peak electric field strength, this created a variety of angle dependent spatial structures in the near-field intensity, beyond approximately the 7th harmonic for the excitation we used. We have also found that in the far-field the propagation smoothed out the intensity and the phase profiles, and the orbital-angular-momenta could be be more clearly observed at the diffraction angle of the far-field intensity maximum.

\section{Acknowledgements}

The project has been supported by the European Union, co-financed by the European Social Fund EFOP-3.6.2-16-2017-00005 - Ultrafast physical processes in atoms, molecules, nanostructures and biological systems It has been also granted by the European Regional Development Fund and the budget of Hungary in the framework of the project GINOP-2.3.2-15-2016-00036 titled Development and application of multimodal optical nanoscopy methods in life and material sciences.

\section{References}

[1] L. Allen, M. W. Beijersbergen et al., Phys Rev. A. 45, 8185 (1992) https://doi.org/10.1103/PhysRevA.45.8185

[2] L. Rego, J. S. Roman et al., Phys. Rev. Lett. 117, 163202 (2016) https://doi.org/10.1103/PhysRevLett.117.163202 
[3] W. Paufler, B. Böning and S. Fritzsche, J. Opt. 21, 094001 (2019) https://doi.org/10.1088/2040-8986/ab31c3

[4] M. Lewenstein, Ph. Balcou et al., Phys Rev A. 49, 2117 (1994) https://doi.org/10.1103/PhysRevA.49.2117

[5] Sz. Majorosi and A. Czirják, Comp. Phys. Comm. 208, 9-28 (2016) https://doi.org/10.1016/j.cpc.2016.07.006 